\title{
Análisis psicométrico del instrumento actitudes hacia las matemáticas mediante el modelo de Respuesta Graduada de Samejima
}

\author{
Psychometric Analysis of Attitudes Toward Mathematics \\ Through the Graduated Response Samejima Model
}

\author{
Olga Rodríguez Jiménez $z^{1}$ \\ Universidad Nacional de Colombia, Colombia \\ Sergio Enrique Mora Mojica² \\ Universidad Nacional de Colombia, Colombia
}

\begin{abstract}
Resumen. La evaluación de las actitudes en el ámbito educativo ha sido utilizada como herramienta para prevenir la pérdida de asignaturas y la deserción académica. El objetivo del estudio es realizar el análisis psicométrico del instrumento Actitudes hacia las Matemáticas con el Modelo de Respuesta Graduada de Samejima (MRG). Se contó con una muestra total de 944 estudiantes de primeros semestres de diferentes carreras universitarias. Se realizó el análisis a partir de la TCT y se ajustó el modelo MRG. Se estimaron y analizaron los parámetros de los ítems y se encontró que la mayoría se ajustan al modelo. En conclusión, se encuentra que la escala cuenta con adecuadas propiedades psicométricas. Finalmente, se presentan las bondades del uso del MRG para la evaluación de actitudes.

Palabras claves. Actitudes, ítems politómicos, Modelo de Respuesta Graduada de Samejima, estudiantes universitarios.

Abstract. The assessment of attitudes in education has been used as a tool for preventing the loss of subjects and the desertion. The aim of the study is to perform a psychometric analysis of the "Attitudes towards Mathematics" scale with Samejima's graded response model (MRG). The sample was of 944 students from different university careers. To analyze the TCT and the MRG model was used. In conclusion, the scale has good psychometric quality. Finally, the benefits of using the MRG for assessing attitudes are presented.
\end{abstract}

Keywords. Attitudes, polytomous items, MRG, college students.

\footnotetext{
${ }^{1}$ Olga Rodríguez Jiménez. Área Curricular de Psicología y Psicoanálisis y Servicio de Atención Psicológica, Universidad Nacional de Colombia, Colombia. Dirección Postal:Cra. 30 \# 45-03, Edificio 212, Oficina 225, Universidad Nacional de Colombia-Sede Bogotá- Sur América. E-mail: orrodriguezj@unal.edu.co

${ }^{2}$ Sergio Enrique Mora Mojica. Universidad Nacional de Colombia. E-mail: semoram@unal.edu.co
}

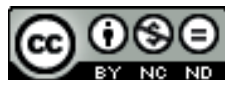

Esta obra está bajo una licencia de Creative Commons Reconocimiento-NoComercial-SinObraDerivada 4.0 Internacional. 


\section{Introducción}

La enseñanza de las matemáticas ha sido fundamental en los planes curriculares de los niveles de educación formal, desde los niveles de enseñanza básica hasta la superior. Las matemáticas son necesarias para el desarrollo de diferentes habilidades intelectuales, ya que se consideran la base del pensamiento racional; por lo que se esperaría que su uso fuera natural para el ser humano y que su aprendizaje se hiciera con gusto y de manera fluida (Maravilla, 2006); lo que en realidad no sucede para ningún nivel de formación.

Considerando que el desarrollo de las matemáticas permite explicar la ocurrencia de diferentes fenómenos naturales, físicos y químicos y de que su aplicación ha sido necesaria para realizar diversos procesos en las ciencias aplicadas y las ciencias humanas (Calderón, 1996; Chamoso \& Rawson, 1998), esta área cuenta con un espacio propio en la mayoría de planes curriculares a nivel universitario. Esto debido a que permite adquirir los cimientos teóricos para entender procesos complejos y aplicados (Álvarez \& Ruiz, 2010), como en el caso de la ingeniería, o porque se asume como herramienta para el análisis de información y la comprensión de procesos lógicos, como en caso de las ciencias humanas.

Pese a lo señalado, los estudiantes presentan, de forma generalizada, un bajo desempeño académico en matemáticas, como lo demuestran los resultados obtenidos por jóvenes de 15 años en las pruebas PISA (OECD, 2012) que están por debajo del promedio respecto a las demás áreas evaluadas y la alta tasa de pérdida de esta asignatura en comparación con otras. Adicionalmente, es una de las áreas de menor interés y agrado entre los estudiantes tanto de educación básica-media como universitaria (Ariza \& Rouquete, 2003; Bazán \& Sotero, 1998; Maravilla, 2006; Mora, 2010; Mato-Vásquez \& De la Torre, 2009). En general, los estudiantes señalan que es difícil, compleja y abstracta (Ernest, 2004, citado por Yavuz Mumcu \& Cansiz Aktas, 2015).

El bajo rendimiento académico en el área ha llevado a buscar factores que expliquen este resultado. En su mayoría, se encuentran factores cognitivos relacionados con el aprendizaje y el análisis de los procesos lógicos que se desarrollan en la asignatura, lo que ha dejado de lado factores de índole emocional, afectivo y actitudinal que pueden interferir con el aprendizaje y uso de las matemáticas (Padron, 2008; Ponce, Martínez \& Zuriaga, 2008). En relación con estos últimos, se ha estudiado tanto el estilo de aprendizaje de cada estudiante, cómo concibe y entiende las matemáticas desde un sentido más abstracto y personal; como también las actitudes y su relación con el desempeño académico. Se ha encontrado una correlación positiva y significativa entre reprobar matemáticas y las actitudes de los alumnos hacia esta área (Castañeda \& Alvarez, 2004) o hacia otras (Mato-Vázquez \& de la Torre, 2009).

Con respecto a la deficiente formación de los estudiantes en el área de matemáticas (Rhodes, 2014) algunas instituciones, para garantizar el éxito en la formación universitaria -ya sea en programas de ingeniería, matemáticas, economía, humanidades (Perlman \& McCann, 1993) o medicina-, han considerado necesario hacer cursos remediales o de nivelación al inicio del proceso educativo. Así, por ejemplo, para 2011 en la Universidad Estatal de California, aproximadamente el $30 \%$ de los estudiantes debieron hacer curso remedial. Un porcentaje similar $(37,6 \%)$ se encuentra para la Universidad de Nevada para el periodo 2006/2007 (Benken, Ramirez, Li \& Wetendorf, 2015). En Latinoamérica, la situación es similar; en la Universidad Nacional de Colombia se realizan cursos de nivelación en matemáticas y lecto-escritura a un porcentaje de estudiantes que va a ingresar a la institución y generalmente oscila entre un 10\% y 30\%.

Estas iniciativas, pertinentes y necesarias, no siempre tienen las consecuencias positivas esperadas, debido a que los cursos tienen un bajo nivel de aprobación, aumentan el tiempo para completar el plan academico, llevan a baja motivación, al abandono antes de finalizarlos y, con ello, generalmente, al abandono de la universidad (Benken, Ramirez, Li \& Wetendorf, 2015). Además, aprobar no garantiza que se adquieren las herramientas necesarias para afrontar cursos posteriores como los relacionados con estadística o metodología. 
Los estudiantes que deben realizar estos cursos, como indican Ho et al. (2000), pueden tener pocas habilidades, actitudes negativas y ansiedad hacia las matemáticas. Como lo señalan Yavuz Mumcu y Cansiz Aktas (2015), la autopercepción, las actitudes, la confianza, las creencias y la ansiedad están vinculadas con la persistencia y motivación para estudiar matemáticas y afectan a esta área más que a otras. Los estudiantes con actitudes positivas muestran más motivación para pensar de forma matemática y comprender el contenido de la clases, también dedican más esfuerzo y trabajo adicional (Kargar, Tarmizi \& Bayat, 2010). Relaciones en este mismo sentido se han encontrado para la geometría (Avc1, Su-Özenir, Özcihan, \& Su, 2014) y la estadística (Escalera-Chávez, García-Santillán \& Venegas-Martínez, 2014).

Por su parte, Yavuz Mumcu y Cansiz Aktas (2015) señalan una asociación positiva entre la autopercepción y las actitudes en matemáticas, y entre estas y los resultados altos en el área, coincidiendo con lo planteado por el National Council of Teachers of Mathematics (2000). Según la entidad, parte del esfuerzo en educación matemática recae en el desarrollo de cambios en la confianza y la disposición de los estudiantes. Esto les permite enfrentar su formación en el área y garantiza un afecto positivo hacia la misma. En conjunto con la alineación curricular entre la educación secundaria y la universitaria, se asegura el conocimiento y las habilidades matemáticas requeridas para enfrentar los estudios superiores (Brown \& Niemi, 2007; Gamoran, Porter, Smithson, \& White, 1997; Hill, 2008; Willett, Hayward, \& Dahlstrom, 2008, citados por Benken, Ramirez, Li \& Wetendorf, 2015).

Pese a reconocer la importancia de los factores afectivos en general y las actitudes en particular, estos frecuentemente se dejan de lado a la hora de plantear propuestas para mejorar el desempeño académico de los estudiantes en el área, además de contar con un menor número de investigaciones. En este sentido, y con el fin de aportar a la adecuada medición y evaluación de las actitudes de los estudiantes hacia las matemáticas, es necesario estudiar las actitudes y su medición, con el fin de contar con instrumentos que puedan ser empleados para orientar y evaluar los programas de apoyo en el área y así contribuir a la asociación positiva entre las dos variables.

Las actitudes cuentan con un buen número de definiciones. Inicialmente, Allport (1935) las consideró como aquello que permite la "relación directa con la experiencia, en donde es un medio que ejerce una influencia dinámica en la respuesta de un individuo a todos los objetos y situaciones con los que se relaciona, ya sea de forma positiva o negativa". Por su parte, Lancheros, Marconi, Manrique y Mendivelso (2007) señalan que la actitud es una "predisposición a responder positiva o negativamente a ciertos objetos o situaciones. Objeto hace referencia a personas, grupos, instituciones, organizaciones y problemas susceptibles de debate. Situaciones se refiere a sucesos o comportamientos de los objetos ya mencionados" (p. 163).

Específicamente, en relación con las actitudes hacia las matemáticas en el campo universitario, Pérez (2008) señala que existen más de 30 definiciones formales de actitud. Algunas de ellas sencillas, como la propuesta por Bem (1972), según la cual, la actitud se concibe como "lo que gusta o lo que no gusta"; otras complejas, como la presentada por Javeau (1978, citado por Pérez, 2008) quien define la actitud como "una manera crónica de vivir la experiencia cotidiana y de responder o rehusar a las solicitudes del mundo, es el "factor de intensidad" de las reacciones psicológicas” (p. 46).

En relación con lo presentado y en busca de una claridad sobre la definición de este constructo y su medición, se entiende que la actitud tiene una direccionalidad, positiva o negativa, la cual se presenta en cierto nivel de intensidad dependiendo de la relación con el objeto en cuestión. Cuando la direccionalidad es positiva, habrá una predisposición al acercamiento, mientras que, cuando su direccionalidad es negativa, naturalmente, existirá una resistencia y reacción contraria a la mencionada. Conceptualmente, la actitud se considera como un constructo unitario que se manifiesta a través de distintos componentes: el afectivo, el cognitivo, el comportamental y el valorativo (García-Santillan et al., 2014; Pérez, 2008). 
El componente afectivo se considera el fundamental de la actitud e incorpora los sentimientos y emociones que despierta en el sujeto el objeto actitudinal. El componente cognitivo comprende concepciones, percepciones, imágenes y creencias que el sujeto tiene sobre el objeto actitudinal. El comportamental explica la forma de actuar del sujeto al manifestar su actitud frente al objeto. El componente valorativo explica la utilidad, valor o ganancia que una determinada actitud puede reportar al sujeto con relación al entorno social, cultural o económico que la persona puede encontrar en el objeto actitudinal (Pérez, 2008). En consecuencia, el estudio y evaluación de las actitudes debe considerar estos componentes.

La evaluación de las actitudes hacia las matemáticas se ha realizado principalmente con cuestionarios con formato de respuesta tipo Likert, siendo uno de los principales instrumentos el desarrollado por Bazán y Sotero (1998), consta de 31 preguntas tipo Likert y está dirigido a estudiantes universitarios de primeros semestres. El trabajo de estos investigadores ha guiado el desarrollo de estudios que tienen como objetivo la evaluación de las actitudes hacia las matemáticas, como primera acción para trabajar en la prevención del bajo rendimiento académico y la deserción con programas de intervención que modifiquen las actitudes frente a la materia (Pérez, 2008; Mora, 2010). De manera complementaria, se encuentra el trabajo realizado por Pineda (2013) en relación con las actitudes hacia la estadística en estudiantes universitarios; reporta que se encuentran mayores actitudes positivas que negativas en las distintas carreras y concluye que el instrumento empleado para medirlas cuenta con las calidades psicométricas adecuadas para ser empleado en evaluación.

El instrumento de Bazán y Sotero fue retomado por Pérez (2008), Preciado (2008) y Mora (2010) para evaluar actitudes en estudiantes universitarios de primer semestre de varias instituciones universitarias en la ciudad de Bogotá, Colombia. Los autores realizaron una revisión teórica y metodológica acerca de la evaluación de actitudes y hallaron que los instrumentos que evalúan actitudes en matemáticas no presentan una revisión sólida de los criterios técnicos de los instrumentos y, por ende, realizan un análisis psicométrico a partir de la Teoría Clásica de los Test (TCT); encontraron adecuados índices de discriminación y dificultad y concluyeron que la prueba en cuestión es fiable.

La teoría clásica de los test es la base de medición psicológica que ha orientado el análisis de instrumentos para evaluar actitudes, aunque es cada vez más frecuente el uso de modelos de Teoría de Respuesta al Ítem (TRI) para dar cuenta de las propiedades psicométricas de los mismos. Ello pese a los requerimientos en tamaño de muestra, a la complejidad de su formulación que afecta su comprensión (Asún \& Zúñiga, 2008), la dificultad para garantizar los supuestos y la dificultad para contar con ítems que hagan una correcta medición a lo largo de toda la escala del constructo (Abal, Lozzia, Aguerri, Galibert \& Attorresi, 2010).

Desde la TCT, los índices de los ítems dependen de las habilidades de la población a la cual se le aplica el instrumento, generando serias limitaciones en cuanto a la interpretación de resultados cuando la prueba se aplica en poblaciones que no son homogéneas (Martínez, 2005). La TRI intenta dar solución a este problema generando estimaciones invariantes de los niveles del atributo evaluado sin depender del instrumento que se utilice o la población que responda el test. Además, presenta ventajas estadísticas que la convierten en un modelo más robusto que brinda un número mayor de indicadores para los ítems y el test.

De acuerdo con Asún y Zúñiga (2008), los modelos TRI cuentan con indicadores que permiten conocer el ajuste del modelo y, con ello, la validez de las estimaciones obtenidas. Además, permiten contar con información precisa sobre la medición de las personas, con lo que es posible conocer qué tan bien el ítem mide a lo largo del continuo de habilidad. Dentro de esta familia de modelos TRI, para el análisis de ítems con respuesta tipo Likert que corresponden a modelos politómicos se cuenta con el modelo de respuesta graduada propuesto por Samejima (1969). 
Según Abad, Ponsoda y Revuelta (2006) este modelo se expresa como:

$$
P^{*}(x \geq k) \cong \frac{1}{1+\exp \left[-\mathrm{Da}\left(\theta-b_{k-1}\right)\right]}
$$

Donde: $\mathrm{D}=$ Constante $; a=\frac{\lambda}{\sqrt{1-\lambda^{2}}} b_{k}=\frac{\tau_{k}}{\lambda}$

En donde el parámetro $a$ hace referencia a la discriminación del ítem e indica la asociación del ítem con el rasgo; $\lambda$ corresponde a la correlación entre $\theta$ y el continuo que se presenta en las categorías de respuesta; este valor oscila entre 0.3 y 2.5 (Abad, Olea, Ponsoda y García, 2011). El parámetro $b_{\mathrm{k}}$ está relacionado con el nivel de $\theta$ en el cual la probabilidad de elegir una categoría o una superior es igual a 0.5 ; y está dada por $\left(b_{(\mathrm{k}-1)}-b_{\mathrm{k}}\right) / 2$ (Abad, Ponsoda y Revuelta, 2006); $\tau_{\mathrm{k}}$ es el punto de corte entre las categorías y corresponde a cada uno de los parámetros $b_{\mathrm{k}}$.

El número de parámetros obtenidos $\left(b_{\mathrm{k}}\right)$ es igual al número de opciones de respuesta menos uno utilizados en la prueba; en este caso, se obtienen 4 parámetros (b1, b2, b3 y b4), los cuales se ubican en una escala continua de -5 a 5 que representa en nivel de habilidad o atributo, en donde valores negativos representan un bajo nivel de atributo, positivos un alto nivel de atributo y valores cercanos a 0 representan un nivel de atributo medio. Es necesario que dentro del planteamiento de los niveles de atributo propuestos mediante las opciones de respuesta, tengan homogeneidad respecto a los niveles de atributo evaluado. La opción más alta representará el nivel de atributo más alto, y la opción de respuesta más baja representará un nivel de atributo bajo.

Luego de la estimación de los parámetros, se hace la comprobación del ajuste al modelo (Muñiz, 1997). Para el modelo de respuesta graduada, siguiendo a LaHuis, Clark y O’Brien (2009), se encuentra que el índice $X^{2} / \mathrm{gl}$ funciona de manera adecuada en una muestra de calibración, de igual forma que la comparación de ítems individuales, pares y triadas a través de este mismo índice.

A partir de lo presentado y con el objetivo de contribuir a consolidar de la evaluación de actitudes hacia un área específica de conocimiento, el presente estudio tiene como objetivo realizar un análisis psicométrico del instrumento Actitudes Hacia las Matemáticas, mediante el modelo de respuesta graduada de Samejima. Se revisa qué ítems se ajustan o no al modelo y si las opciones de respuesta planteada logran diferenciar entre niveles de habilidad. De esa manera se puede aportar un instrumento con adecuadas características psicométricas que garanticen su uso en procesos de diagnóstico o clasificación.

\section{Método}

\section{Participantes}

Con un muestreo intencional, se contó con la participación de 944 estudiantes de primer semestre pertenecientes a los programas de Psicología (282), Trabajo Social (82), Contaduría Pública (21), Administración de Empresas (18) e Ingeniería (436) de tres universidades de la ciudad de Bogotá y una de la ciudad de Popayán. Las carreras se seleccionaron según el número de asignaturas relacionadas con matemáticas que se incluyen en los pensum, para abarcar diferentes niveles de desempeño académico y actitudinal en el área. Las universidades fueron seleccionadas según los resultados obtenidos en las pruebas de estado para el ingreso a la educación superior SABER PRO según los rangos de alto, medio y bajo y la facilidad de acceso a las instituciones educativas. En los dos primeros niveles se contó únicamente con la participación de las carreras de Psicología e Ingeniería. Para el análisis se excluyeron 105 casos dado que no respondieron alguna de las preguntas en la prueba de actitud o marcaron más de una opción de respuesta. La muestra total considerada en el análisis fue de 839 estudiantes, 444 mujeres y 391 hombres, 4 estudiantes no marcaron la hoja de respuestas, por lo que fue imposible identificar su género.

\section{Instrumentos}

El instrumento objeto de análisis psicométrico 
se denomina "Actitudes Hacia las Matemáticas" de Mora (2010), modificado y ajustado por Pérez (2008), Preciado (2008) y Mora (2010) del original diseñado por Bazán y Sotero en 1998. El instrumento consta de 21 afirmaciones de respuesta graduada tipo Likert y se debe evaluar el nivel de acuerdo o desacuerdo frente a cada una de estas, en una escala que va desde "Totalmente en desacuerdo" hasta "Totalmente de acuerdo". La calificación es ordinal de 1 a 5 dependiendo de la direccionalidad hacia las matemáticas (Anexo 1). La prueba está compuesta por afirmaciones que dan cuenta de los componentes afectivo, cognitivo, valorativo y comportamental (Lancheros et al., 2007; Pérez, 2008; Preciado, 2008).

El instrumento diferencia entre actitudes altas, medias y bajas hacia las matemáticas según las puntuaciones obtenidas. Los estudiantes que están en el rango inferior de puntajes se consideran con baja actitud hacia las matemáticas y se caracterizan por tener desagrado, desinterés y darle poco sentido al aprendizaje de la misma. En contraposición, los estudiantes con mayor puntuación y, en consecuencia, con actitudes altas, presentan agrado, interés y motivación para estudiar matemáticas, considerándolas un elemento importante para su desarrollo profesional. Los estudiantes cuyas puntuaciones están cerca del promedio son considerados con un nivel de actitud medio, mostrando interés por las matemáticas, pero no motivación por el aprendizaje de ellas; su comportamiento está ligado al cumplimiento de las labores académicas. En el análisis se hace referencia al nivel de actitud en función del continuo de la habilidad, medida que se obtiene a partir del análisis con modelos TRI.

\section{Procedimiento}

El instrumento se aplicó durante la jornada académica, en la mayoría de casos, en la hora correspondiente a la asignatura de matemáticas. Las condiciones del ejercicio permitieron la correcta y tranquila aplicación del instrumento.

Para el análisis psicométrico se consideraron los siguientes procedimientos:

i)Análisis de indicadores a partir de la TCT.
-Análisis de las frecuencias por opción para cada ítem de la prueba de actitudes y análisis de discriminación mediante el método de correlación ítem-test (valores superiores a 0.2 indica que el ítem discrimina).

-Evaluación del índice de frabilidad por medio del coeficiente $\alpha$ de Cronbach, que indica el grado en que las medidas obtenidas dependen de los ítems aplicados (Abad, Olea, Ponsoda y García, 2011).

ii) Comprobación de supuestos.

-El primer supuesto evaluado correspondió a la unidimensionalidad; entendida como la probabilidad que un individuo tiene de responder de cierta manera en un ítem depende únicamente de su nivel de habilidad en lo evaluado en el test. Se evaluó por medio del análisis factorial dado que, según Abad et al. (2011), es el más indicado para el análisis de datos categóricos, debido a que asume continuidad en las variables y la existencia de una relación lineal entre la variable latente y los ítems.

-El segundo supuesto es el de la independencia local; hace referencia al hecho de que la respuesta de un sujeto con determinado valor de habilidad en una variable unidimensional no es inducida por otros ítems con el mismo nivel de atributo. Para la evaluación de este supuesto se compararon los ítems de forma individual, por pares y triadas mediante el índice $X^{2} / \mathrm{gl}$ (Drasgow, Levine, Tsien, Williams y Mead (1985); RodríguezJiménez, 2011).

La muestra total se dividió en dos con la intención de corroborar mediante el análisis factorial exploratorio (AFE) y análisis factorial confirmatorio (AFC) la unidimensionalidad, utilizando una de las mitades elegidas de forma aleatoria para cada uno de los dos procesos.

En las tablas 1 y 2 se observa la información demográfica de los estudiantes para las dos submuestras, corroborando la similaridad en cuanto a género y programa de estudios de los participantes.

Análisis factorial exploratorio (AFE). Se utilizó el método de análisis factorial exploratorio para evaluar el ajuste de la escala y partir de ello para proponer un modelo unidimensional a corroborar mediante el 
AFC. Se manejó el método de mínimos cuadrados generalizados, debido a que utiliza una prueba de bondad de ajuste para evaluar si el modelo factorial se ajusta a las correlaciones o covarianzas obtenidas en la matriz de análisis, además de que no exige el cumplimiento del supuesto de normalidad para poderse ejecutar (Ximénez, 2010).

Análisis factorial confirmatorio (AFC). Busca enmarcar dentro de un modelo ya establecido la relación de los ítems dentro de los factores, revisando la existencia de correlaciones y covarianzas entre estos últimos. Para el análisis factorial confirmatorio, fue necesario identificar el modelo mediante la matriz de correlaciones y covarianzas de los elementos que componen el modelo. Se establecieron los pesos factoriales a 1 para identificar el modelo (Abad et al., 2011). Se utilizó el método de mínimos cuadrados no ponderados (ULS) debido a la ausencia de distribución normal multivariada. Se tomó como referencia el estadístico del índice de bondad de ajuste RMR (análisis de residuos) y el SMRS, que es el índice RMR ajustado, estos constituyen un indicador del ajuste de los ítems al modelo propuesto. Por su parte,el índice NFI (NormedFitFixed) informa sobre el grado de ajuste del modelo propuesto en contraste con otro modelo alternativo (Abad et al., 2011; Schreiber, Stage, King, Nora \& Barlow, 2006).

El criterio para indicar un adecuado ajuste al modelo según cada método de estimación es el siguiente: para el RMR valores cercano a 0 (Hu y Bentler, 1988 citado en Abad et al., 2011) y menores a 0.08; para la medida de ajuste SMRM valores inferiores a 0.08; para el ajuste comparativo por NFI un valor superior o igual a 0.95 .

Tabla 1

Género de los participantes por submuestra de análisis

\begin{tabular}{cccc}
\hline Género & AFE & AFC & Total \\
\hline Masculino & 186 & 205 & 391 \\
Femenino & 232 & 212 & 444 \\
\hline Total & 420 & 419 & 839 \\
\hline
\end{tabular}

Tabla 2

Programa académico de los participantes por submuestra de análisis

\begin{tabular}{cccc}
\hline Programa & AFE & AFC & Total \\
\hline Psicología & 145 & 137 & 282 \\
Trabajo Social & 37 & 45 & 82 \\
Administración & 10 & 8 & 18 \\
Contaduría & 14 & 7 & 21 \\
Ingeniería & 214 & 222 & 436 \\
\hline Total & 420 & 419 & 839 \\
\hline
\end{tabular}


Estimación de parámetros con el modelo de respuesta graduada de Samejima (MRG). En consideración de que es el método más utilizado para evaluar test con ítems de más de dos categorías de respuesta organizadas de forma ordinal desde el enfoque de la Teoría de respuesta a los ítems (Rodríguez-Jiménez, 2011). El ajuste se evaluó con el índice $X^{2} / \mathrm{gl}$ luego de haber estimado los parámetros según lo señalado por Drasgow, Levine, Tsien, Williams y Mead (1985).

\section{Análisis de datos}

Se utilizó el programa EXCEL 2013 para obtener las distribuciones y procesos de análisis clásico. Los análisis de fiabilidad por $\alpha$ de Cronbach, Pearson y análisis factorial exploratorios se realizaron mediante el software SPSS 21. El análisis factorial confirmatorio se llevó a cabo en el programa AMOS 21 (Arbuckle, 2012) y la estimación de los parámetros y el ajuste al modelo TRI en los programas MULTILOG 7.0 (Scientific software International, Inc, 2003) y MODFIT 6-06-01 web (Stark, 2001).

\section{Resultados}

\section{Indicadores de la Teoría clásica de los Test}

En la Tabla 3, se presenta para cada uno de los ítems, la puntuación media, las distribuciones en los diferentes niveles de respuesta y el nivel de discriminación. El ítem con mayor puntuación promedio fue el 16 (Estudiar matemáticas me hace perder tiempo valioso); en contraste con los ítems 3 (Me agrada tener que abordar muchos cursos de matemáticas), 5 (Debo estudiar matemáticas si quiero ser profesional, pero las matemáticas no son mi asignatura favorita), 14 (Solo deberían enseñarse en matemáticas las cosas prácticas que utilizaremos como profesionales) y 21 (Las matemáticas son mi asignatura favorita), que presentan los promedios de puntuaciones más bajos. (Tabla 3 )

En general, los ítems presentan un buen nivel de discriminación, tomando como referencia el criterio expresado por Schmeiser y Welch (2006) en donde valores menores a 2 nos llevarían a descartar el ítem, por lo que ninguno de los ítems fue eliminado. El ítem con más bajo nivel de discriminación fue el 15 y los más altos el 7 y el 12. En relación con la fiabilidad, se encuentra el índice Alpha de Cronbach igual a .934.

Análisis factorial exploratorio (AFE)

Se encuentra que el modelo ajusta $(\mathrm{p}=.103)$ con un porcentaje de varianza explicada de 51.06\%. En consecuencia, se considera que existe un único factor que representa el constructo denominado actitud hacia las matemáticas. El peso de cada ítem en el factor se presenta en la tabla 3.

\section{Análisis factorial confirmatorio (AFC)}

A partir de la estructura factorial definida en el AFE, se representó la estructura factorial para el instrumento (Figura 1). Se ajusta el peso de una variable de cada factor a 1, para que el número de grados de libertad fuera positivo, el método empleado fue ULS, debido a la no normalidad multivariada de los datos utilizados. En relación con el ajuste al modelo, se tiene un índice SRMR $=0,059$, un índice RMR $=0,080$ y un índice NFI $=0,979$. Según los criterios señalados, es correcto afirmar que el modelo se ajusta a la estructura propuesta. Se evidencia que existe un solo factor que se trata de la actitud hacia las matemáticas y, en consecuencia, se procede a estimar los parámetros correspondientes en TRI (Figura 1).

Estimación de parámetros con el modelo de respuesta graduada de Samejima

Se estimaron los parámetros para el modelo de respuesta graduada (MRG) acorde con el ajuste a este modelo. En la prueba de razón de verosimilitud se consideran las hipótesis: Ho: los datos se ajustan al modelo de MRG-a constante; Ha: los datos se ajustan al modelo MRG. Los resultados permiten concluir que el modelo MRG tiene mejor ajuste, con un nivel de significación de $0.00\left(X^{2}=800,9 ; \mathrm{gl}=20\right)$.

Para su interpretación, es necesario aclarar que las opciones de respuesta tienen una distribución homogénea respecto a los parámetros. Un valor menor que el presentado para el parámetro b1 señala que la opción de respuesta evalúa el menor nivel de habilidad. Los valores entre los parámetros b1 y b2 señalan opciones que miden niveles medio-bajos de habilidad. 
Tabla 3

Descriptivos de los items: promedio, indice de discriminación y porcentaje de respuesta por opción y Análisis Factorial Exploratorio

Frecuencia por opción de respuesta

\begin{tabular}{ccccccccc}
\hline Ítem & Media & Discriminación & $\begin{array}{c}1 \\
\%\end{array}$ & $\begin{array}{c}2 \\
\%\end{array}$ & $\begin{array}{c}3 \\
\%\end{array}$ & $\begin{array}{c}4 \\
\%\end{array}$ & $\begin{array}{c}\text { Peso en el factor } \\
\text { (AFE) }\end{array}$ \\
\hline 1 & 3.619 & .64 & 5.24 & 15.26 & 7.99 & 55.42 & 16.09 & .639 \\
2 & 3.207 & .67 & 7.39 & 23.96 & 18.36 & 41.12 & 9.18 & .794 \\
3 & 2.889 & .62 & 11.56 & 31.47 & 20.38 & 29.68 & 6.91 & .729 \\
4 & 3.360 & .50 & 7.63 & 20.62 & 18.59 & 34.45 & 18.71 & .412 \\
5 & 2.801 & .60 & 22.53 & 27.29 & 9.89 & 28.13 & 12.16 & .553 \\
6 & 3.265 & .68 & 10.97 & 19.31 & 14.54 & 42.67 & 12.51 & .687 \\
7 & 3.143 & .78 & 7.39 & 26.70 & 18.00 & 40.05 & 7.87 & .786 \\
8 & 3.244 & .56 & 6.08 & 25.03 & 18.12 & 39.93 & 10.85 & .560 \\
9 & 3.081 & .74 & 7.15 & 28.37 & 23.12 & 31.94 & 9.42 & .752 \\
10 & 3.493 & .63 & 5.13 & 17.16 & 16.45 & 45.77 & 15.49 & .419 \\
11 & 3.514 & .72 & 4.29 & 18.36 & 13.83 & 48.75 & 14.78 & .544 \\
12 & 3.322 & .78 & 12.51 & 17.04 & 13.59 & 39.45 & 17.40 & .605 \\
13 & 3.732 & .52 & 3.81 & 12.16 & 11.56 & 51.97 & 20.50 & .294 \\
14 & 2.876 & .57 & 20.50 & 23.96 & 16.33 & 25.86 & 13.35 & .311 \\
15 & 3.386 & .49 & 5.60 & 19.19 & 22.29 & 36.83 & 16.09 & .405 \\
16 & 4.142 & .50 & 1.79 & 3.22 & 8.34 & 52.32 & 34.33 & .337 \\
17 & 3.366 & .73 & 6.32 & 20.50 & 19.31 & 38.02 & 15.85 & .569 \\
18 & 3.054 & .76 & 6.79 & 31.35 & 20.38 & 32.66 & 8.82 & .638 \\
19 & 3.203 & .52 & 8.34 & 23.12 & 17.04 & 42.91 & 8.58 & .345 \\
20 & 3.700 & .67 & 3.46 & 10.85 & 15.26 & 53.16 & 17.28 & .480 \\
21 & 2.723 & .76 & 16.92 & 30.51 & 23.84 & 20.74 & 7.99 & .589 \\
\hline Promedio & 3.349 & .637 & 8.196 & 20.342 & 15.511 & 40.290 & 15.662 & \\
\hline
\end{tabular}

Valores entre los parámetros b2 y b3, opciones que evalúan niveles medios de habilidad. Valores entre los parámetros b3 y b4, la opción que representa niveles medio- altos de habilidad y valores superiores a b4, la opción que representa un nivel alto de habilidad. Es importante anotar que la habilidad hace referencia al atributo medido que, para el caso, se trata de actitud.

En la tabla 4, se presentan los parámetros estimados; se aprecia que los ítems con el parámetro a (discriminación) más alto son el 2 (2.74) "Disfruto con los ejercicios y problemas de matemáticas", el 7 (2.77) "Me siento seguro al trabajar en matemáticas", el $9(2,62)$ "La carrera que voy a estudiar tiene matemáticas, y eso me encanta", el 18 (2.95) "Cuando estudio matemáticas, el tiempo se me pasa muy entretenido y rápido" y el 21 (2.84) “Las matemáticas 


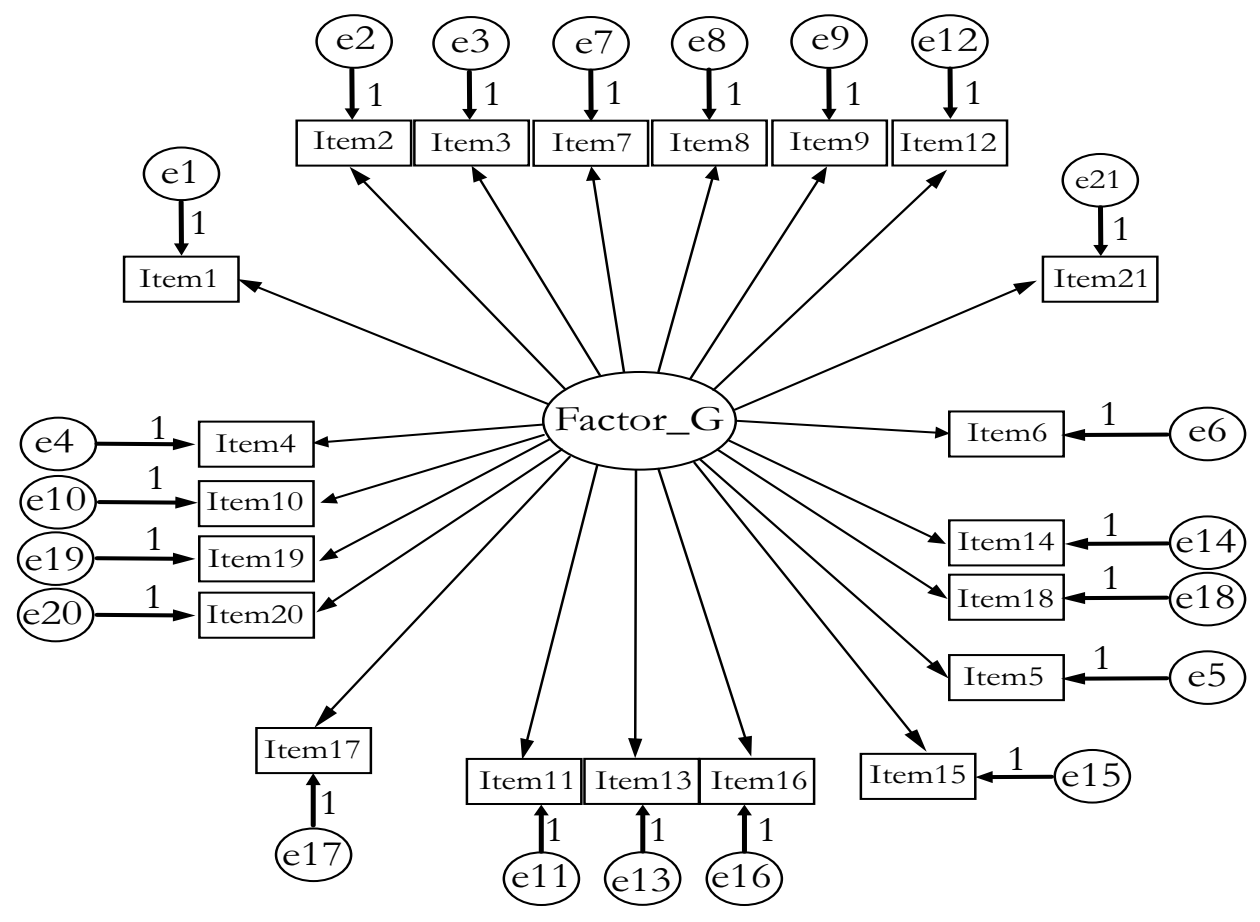

Figura 1. Representación gráfica del modelo factorial del instrumento de actitudes hacia las matemáticas

son mi asignatura favorita". Todos en contraposición con los ítems 13 (1.07) "Mi mente se pone en blanco y soy incapaz de pensar claramente cuando hago matemáticas", 14 (1.26) "Solo deberían enseñarse en matemáticas las cosas prácticas que utilizaremos como profesionales", 15 (1.13) "Me agradaría, si es necesario, tomar más cursos de matemáticas" y 19 (1.1) "Se me dificulta comprender y manejar el lenguaje matemático", los cuales presentan un índice bajo de discriminación. Los ítems con un mayor nivel de discriminación permiten evaluar mejor los niveles de habilidad de actitudes hacia las matemáticas.

La diferencia en valor absoluto entre los parámetros $b_{\mathrm{k}}$ indica la probabilidad de que esa opción sea elegida en un determinado continuo de habilidad $\theta$. Como se aprecia en la Tabla 4, la diferencia promedio más alta se presenta entre los parámetros b3 y b4 y la diferencia más pequeña fue la de los parámetros b2 y b3. En este rango tres ítems presentan puntajes mayores a 1 en la diferencia. La opción de respuesta que representa niveles medios de habilidad (no sabe o no puede contestar) puede no estar diferenciando entre la opción de nivel medio- alto de habilidad, dado que el parámetro $b_{k}$ en el continuo de habilidad para las dos opciones indica que están evaluando niveles de rasgo similares.

En la revisión de los valores máximos y mínimos de los parámetros para cada uno de los ítems, se encontró que el ítem en el que es más fácil estar "totalmente de acuerdo" cuando implica un alto nivel de atributo fue el 19 "Se me dificulta comprender y manejar el lenguaje matemático". El ítem en el que es más difícil estar "totalmente de acuerdo" cuando implica un alto nivel de habilidad fue el 12 "Prefiero estudiar cualquier otra materia en lugar de matemáticas". De forma contraria, 
el ítem en el que es más fácil responder "totalmente en desacuerdo" cuando se refiere a un bajo nivel de atributo fue el 21 "Las matemáticas son mi asignatura favorita" y el ítem en el que es más difícil responder "totalmente en desacuerdo" cuando implica un bajo nivel de habilidad fue el 16 "Estudiar matemáticas me hace perder tiempo valioso".

En la función de información para cada ítem (Anexo 2) se observa que los ítems que resultan menos informativos en el continuo de atributo $(\theta)$ son
$4,5,8,10,13,14,15,16,19$ y 20 , siendo los ítems 4 $(0,24)$ y $16(0,24)$ los que tienen mayor nivel de error promedio en sus parámetros. Cuanto mayor sea el error, menor es el nivel de información que brindan los ítems en los distintos niveles de habilidad. En cuanto a los errores de medida, los parámetros b1 y b4 presentan los mayores valores promedio de 0.178 y 0.13 respectivamente. El parámetro b3 presenta el menor nivel de error con un promedio de 0,079. Esto concuerda con que, a nivel general, el error explicado en la prueba de los niveles medios de atributo tiene un

Tabla 4

Parámetros de los items para el modelo de respuesta graduada de Samejima

\begin{tabular}{|c|c|c|c|c|c|c|c|c|}
\hline Ítem & A & $\mathrm{b} 1$ & $\mathrm{~b} 1-\mathrm{b} 2$ & $\mathrm{~b} 2$ & b2-b3 & b3 & b3-b4 & b4 \\
\hline \multirow{2}{*}{1} & 1.69 & -2.26 & -1.19 & -1.07 & -0.38 & -0.69 & -2.2 & 1.51 \\
\hline & (.11) & $(.18)$ & - & $(.09)$ & - & $(.08)$ & - & (.11) \\
\hline \multirow{2}{*}{2} & 2.74 & -1.62 & -1.17 & -0.45 & -0.58 & 0.13 & -1.51 & 1.64 \\
\hline & (.16) & $(.09)$ & - & $(.06)$ & - & $(.05)$ & - & $(.09)$ \\
\hline \multirow{2}{*}{3} & 2.13 & -1.44 & -1.35 & -0.09 & -0.67 & 0.58 & -1.43 & 2.01 \\
\hline & $(.12)$ & $(.09)$ & - & $(.06)$ & - & $(.06)$ & - & (.13) \\
\hline \multirow{2}{*}{4} & 0.88 & -3.09 & -1.96 & -1.13 & -1.07 & -0.06 & -2.08 & 2.02 \\
\hline & (.1) & $(.36)$ & - & $(.17)$ & - & $(.12)$ & - & $(.24)$ \\
\hline \multirow{2}{*}{5} & 1.44 & -1.06 & -1.14 & 0.08 & -0.39 & 0.47 & -1.46 & 1.93 \\
\hline & (.1) & (.1) & - & $(.08)$ & - & $(.08)$ & - & (.16) \\
\hline \multirow{2}{*}{6} & 1.78 & -1.62 & -1.03 & -0.59 & -0.54 & -0.05 & -1.74 & 1.69 \\
\hline & $(.11)$ & $(.12)$ & - & $(.08)$ & - & $(.07)$ & - & $(.12)$ \\
\hline \multirow{2}{*}{7} & 2.77 & -1.62 & -1.23 & -0.39 & -0.55 & 0.16 & -1.57 & 1.73 \\
\hline & $(.16)$ & (.1) & - & $(.05)$ & - & $(.05)$ & - & $(.09)$ \\
\hline \multirow{2}{*}{8} & 1.43 & -2.37 & -1.68 & -0.69 & -0.73 & 0.04 & -1.95 & 1.99 \\
\hline & (.1) & $(.2)$ & - & $(.09)$ & - & $(.08)$ & - & (.16) \\
\hline \multirow{2}{*}{9} & 2.62 & -1.66 & -1.32 & -0.34 & -0.71 & 0.37 & -1.26 & 1.63 \\
\hline & $(.14)$ & (.1) & - & $(.06)$ & - & $(.05)$ & - & $(.09)$ \\
\hline \multirow{2}{*}{10} & 1.51 & -2.42 & -1.44 & -0.98 & -0.71 & -0.27 & -1.9 & 1.63 \\
\hline & $(.12)$ & $(.2)$ & - & (.1) & - & $(.07)$ & - & (.13) \\
\hline \multirow{2}{*}{11} & 2.25 & -2.09 & -1.28 & -0.81 & -0.54 & -0.27 & -1.67 & 1.4 \\
\hline & $(.13)$ & $(.14)$ & - & $(.07)$ & - & $(.06)$ & - & $(.09)$ \\
\hline \multirow{2}{*}{12} & 2.48 & -1.33 & -0.79 & -0.54 & -0.48 & -0.06 & -1.27 & 1.21 \\
\hline & $(.14)$ & $(.08)$ & - & $(.07)$ & - & $(.05)$ & - & $(.07)$ \\
\hline
\end{tabular}

Continúa... 


\begin{tabular}{|c|c|c|c|c|c|c|c|c|}
\hline Ítem & A & b1 & b1-b2 & $\mathrm{b} 2$ & b2-b3 & b3 & b3-b4 & b4 \\
\hline \multirow[t]{2}{*}{13} & 1.07 & -3.37 & -1.63 & -1.74 & -0.75 & -0.99 & -2.61 & 1.62 \\
\hline & (.1) & $(.36)$ & - & (.19) & - & (.13) & - & (.17) \\
\hline \multirow[t]{2}{*}{14} & 1.26 & -1.28 & -1.17 & -0.11 & -0.68 & 0.57 & -1.4 & 1.97 \\
\hline & $(.11)$ & (.13) & - & $(.09)$ & & $(.09)$ & - & (.18) \\
\hline \multirow[t]{2}{*}{15} & 1.13 & -2.84 & -1.78 & -1.06 & -1.11 & 0.05 & -1.86 & 1.91 \\
\hline & (.1) & $(.27)$ & - & (.13) & - & (.09) & - & (.19) \\
\hline \multirow[t]{2}{*}{16} & 1.12 & -4 & -1.01 & -2.99 & -1.09 & -1.9 & -2.73 & 0.83 \\
\hline & $(.11)$ & $(.47)$ & - & $(.31)$ & - & $(.19)$ & - & (.12) \\
\hline \multirow[t]{2}{*}{17} & 2.17 & -1.86 & -1.19 & -0.67 & -0.66 & -0.01 & -1.36 & 1.35 \\
\hline & (.13) & $(.12)$ & - & $(.07)$ & - & $(.06)$ & - & $(.09)$ \\
\hline \multirow[t]{2}{*}{18} & 2.95 & -1.64 & -1.42 & -0.22 & -0.61 & 0.39 & -1.24 & 1.63 \\
\hline & $(.16)$ & $(.1)$ & - & $(.05)$ & - & $(.05)$ & - & $(.08)$ \\
\hline \multirow[t]{2}{*}{19} & 1.1 & -2.48 & -1.73 & -0.75 & -0.8 & 0.05 & -2.6 & 2.65 \\
\hline & $(.09)$ & $(.25)$ & - & $(.12)$ & - & $(.1)$ & - & $(.25)$ \\
\hline \multirow[t]{2}{*}{20} & 1.78 & -2.48 & -1.11 & -1.37 & -0.77 & -0.6 & -2.01 & 1.41 \\
\hline & (.13) & $(.2)$ & - & $(.1)$ & - & $(.08)$ & - & (.1) \\
\hline \multirow[t]{2}{*}{21} & 2.84 & -1.03 & -1.07 & 0.04 & -0.71 & 0.75 & -0.95 & 1.7 \\
\hline & $(.15)$ & $(.07)$ & - & $(.05)$ & - & $(.05)$ & - & $(.09)$ \\
\hline \multirow{2}{*}{ Promedio } & 1.864 & -2.07 & -1.32 & -0.76 & -0.69 & -0.06 & -1.75 & 1.69 \\
\hline & $(.122)$ & (.178) & - & (.1) & - & $(.079)$ & - & (.13) \\
\hline
\end{tabular}

Nota. Entre parentesis se encuentra el error estándar

menor nivel de error, contrario a los niveles extremos que presentan un mayor nivel de error. En cuanto a la función de información general, como se observa en la figura 2, la prueba brinda una mayor información para niveles de atributo entre $-2 \mathrm{y}+2$.

\section{Ajuste de los items}

Mediante la comparación por diadas y triadas, el índice de $X^{2} / \mathrm{gl}$ encontró que 12 ítems mostraron valores menores a 2 , dos ítems valores menores a 3 , dos ítems con valores menores a 4 y cinco ítems con valores mayores a 7 . Teniendo en cuenta los criterios dados por Chernyshenko, Stark, Chan, Drasgow y Williams (2001), valores menores a 4 son aceptables, y superiores a 4 inaceptables, es decir, que la mayoría de ítems se ajustan al modelo. Adicionalmente, y en atención a que este es el mismo indicador para comprobar el supuesto de independencia local, se considera que para estos datos se cumple con este supuesto, dado que los autores señalan que no presentar ajuste se puede deber a que los datos son multidimensionales o no independientes.

\section{Discusión y conclusiones}

El análisis realizado presenta las características psicométricas del instrumento Actitudes Hacia las Matemáticas. Los resultados obtenidos en el AFE y AFC demuestran la existencia de un modelo unifactorial y una organización de los ítems consistente con la organización teórica propuesta por Mora (2010). Además, presenta un índice de consistencia interna alto, lo que indica que las respuestas dadas por los estudiantes se deben a características que evalúa la prueba y no a elementos externos. Los resultados evidencian el ajuste al modelo MRG donde los 


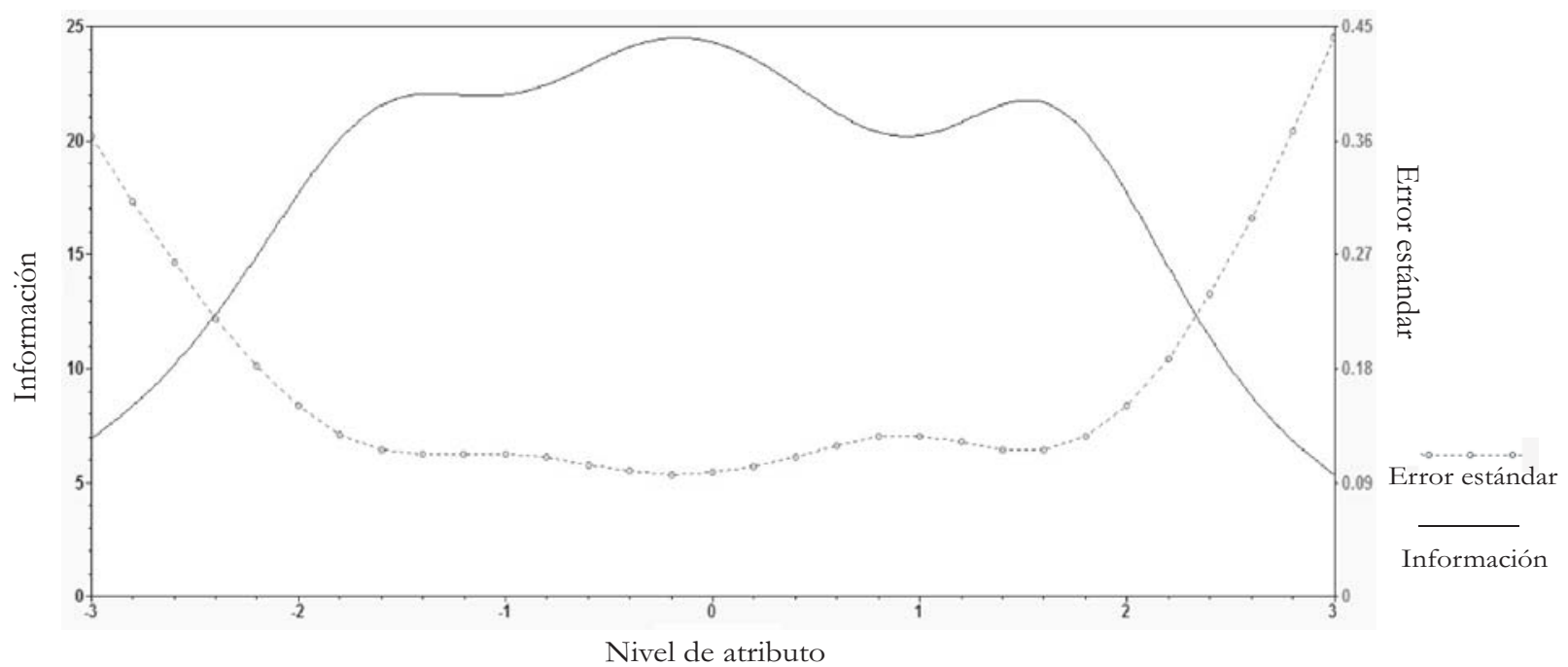

Figura 2. Función de información y error estándar para el test

parámetros son independientes.

Los resultados obtenidos de los errores estándar de los parámetros indican que la prueba evalúa mejor a los estudiantes quienes en el continuo de habilidad $(\theta)$ evidencian niveles medios y altos, ya que los niveles de error promedio son menores en los parámetros b2, b3 y b4. Para el menor nivel de habilidad (b1), el nivel de error es en promedio mayor que los demás niveles. Esto se corrobora en la gráfica de función de información del test y de cada uno de los ítems, donde los ítems con un parámetro b1 menor a -2 y mayores a 2,5 presentan, en general, un mayor nivel de error en cuanto a la información presentada para la evaluación de niveles menores y mayores de habilidad. Para el instrumento total, se tiene que en el continuo de habilidad se evalúa mejor los niveles medio y alto, los niveles muy bajos de habilidad son evaluados con un mayor nivel de error, por lo que es necesario revisar la pertinencia de contar con preguntas cuyos parámetros b1 son inferiores a -2 .

Se recomienda revisar los ítems 13, 14, 15 y 19 que tienen baja discriminación y un valor bajo en la función de información. Por su parte, se destacan los ítems 2, 3, 7, 9, 12, 18 y 21, dado que sus parámetros presentan una distribución adecuada en sus opciones de respuesta y en los niveles de habilidad que pretenden evaluar y con lo cual miden mejor las actitudes hacia las matemáticas. Adicionalmente, para estos ítems las opciones de respuesta se distribuyen de acuerdo con lo esperado en el modelo, evidenciando que cada opción mide adecuadamente en el continuo de habilidad y, por ende, son opciones posibles.

Se encuentra que el instrumento cuenta con ítems para evaluar los distintos niveles de actitud; sin embargo, para algunos será necesario eliminar la opción de respuesta intermedia para optimizar el posicionamiento de los estudiantes dentro de la escala de actitudes hacia las matemáticas. A partir de la función de información del test, se tiene que la escala sirve para evaluar las actitudes hacia las matemáticas; la curva de función de información en el test total arroja valores altos para todos los niveles de atributo en particular entre - 2 y 2 y niveles bajos de error, así, se considera que la prueba es confiable para la evaluación 
de actitudes a nivel universitario.

En relación con el índice de ajuste $X^{2} / g l$ se tiene que funcionó de manera adecuada para evaluar el ajuste de los ítems, siendo destacable el hecho de reportar baja tasa de error tipo I y mayor poder (LaHuis, Clark \& O’Brien, 2009); aunque no del todo deseables para evaluar la independencia local, dado que se evalúa con posterioridad a la obtención de los parámetros de los ítems. De acuerdo con Abad, Olea, Ponsoda y García (2011), esto pone en evidencia una de las desventajas de los modelos TRI: "los procedimientos para determinar el ajuste de los modelos no son totalmente satisfactorios" (p.156).

En relación con el uso de métodos para el análisis de ítems categóricos desde la TRI, se concluye que son de gran valor al momento de evaluar una prueba de actitudes (Asún \& Zúñiga, 2008), debido a que en contraste con los resultados obtenidos con la TCT, el MRG informa sobre el comportamiento de cada uno de los ítems a través de las funciones de respuesta para las categorías, con lo cual se puede conocer el funcionamiento de cada una de estas acorde con el nivel de habilidad requerido para elegirla, esto permite decidir sobre la pertinencia de tener en cuenta o no una categoría de respuesta. En este sentido, los datos analizados respaldan estudios previos que afirman que las opciones intermedias son poco informativas (González-Romá \& Espejo, 2003; Hernández, Espejo \& González-Romá, 2006) y que cuatro alternativas de respuesta son sus suficientes para modelos de TRI politómicos (Gray-Litter, Williams \& Hancock, 1997; Hernández et al., 2000).

Se recomienda el uso del MRG para el análisis en la evaluación de actitudes en particular o en general, para pruebas que usen una escala de respuesta graduada. Estos modelos permiten realizar un análisis detallado del contenido de los ítems en función de sus parámetros de localización y discriminación, lo que contribuye a mejorar la calidad del instrumento, ya que se pueden agregar afirmaciones que sean más informativas en segmentos específicos del continuo actitudinal. Por otra parte, contar con la función de información para cada ítem y la escala total permite conocer la precisión con la cual se mide en el continuo de habilidad.

Finalmente, en relación con el uso de estos modelos, se destaca la facilidad para la interpretación gráfica de cada parámetro y la precisión en la medida, a través de las curvas de funcionamiento de los ítems por categoría y las curvas de función de información por ítem y por test.

Por todo lo anterior, se concluye que el instrumento Actitudes hacia las Matemáticas cuenta con adecuadas calidades psicométricas, por lo que puede ser usado en los procesos de medición de los programas remediales relacionados con el aprendizaje de las matemáticas que adelantan las universidades.

\section{Referencias}

Abad, F., Ponsoda, V., \& Revuelta, J. (2006). Modelos politómicos de respuesta al item. Madrid: La Muralla

Abad, F., Olea, J., Ponsoda, V., \& García, C. (2011). Medición en ciencias sociales y de la salud. Madrid: Editorial Síntesis.

Abal, F., Lozzia, S. G., Aguerri, M., Galibert, M., \& Attorresi, H. (2010). La escasa aplicación de la teoría de respuesta al ítem en tests de ejecución típica. Revista Colombiana de Psicología, 19, 111-122.

Allport, G. W. (1935). Attitudes. En: Handbook of social psychology. Worcester: Clark University Press.

Álvarez, Y., \& Ruiz, M. (2010). Actitudes hacia las matemáticas en estudiantes de ingeniería en universidades autónomas venezolanas. Revista de Pedagogía, 31(89), 225-249.

Arbuckle, J. (2012). IBM® SPSS® Amos ${ }^{\mathrm{TM}} 21$ User's Guide. Amos Development Corporation.

Ariza, E., \& Rouquette, J. (2003). Análisis de factores que influyen en el aprendizaje de las matemáticas mediante un laboratorio virtual. México: Ed. Universidad Autónoma Metropolitana, unidad Xochimilco.

Asún, R., \& Zúñiga, C. (2008). Ventajas de los modelos politómicos de Teoría de Respuesta al Ítem en la medición de actitudes sociales. El análisis de un 
caso. PSYKHE, 17(2), 103-115.

Avc1, E., Su-Özenir, Ö., Özcihan, H.G., \& Su, G. (2014). Attitudes of high school students towards geometry. Turkish Journal of Computer and Mathematics Education, 5(3), 304-317.

Bazán, J. L., \& Sotero, H. (1998). Una aplicación al estudio de actitudes hacia la matemática en la UNALM. Revista Anales Cientificos. UNALM, 36 (1), 60-72.

Bem, D. J. (1972). Selfperception theory. Journal for Advances Experimental social psychology, 6, 2-62.

Benken, B., Ramirez, J., Li, X., \& Wetendorf, S. (2015). Developmental Mathematics Success: Impact of Students' Knowledge and Attitudes. Journal of developmental education, 38(2), 14-31.

Calderón, A. (1996). Investigación y didácticas de las matemáticas. Madrid: Ed Narcea.

Castañeda, A., \& Alvarez, M. J. (2004). La reprobación en Matemáticas. Dos experiencias. Tiempo de Educar. Revista Interinstitucional de Imvestigación Educativa, 5(9), 141-172.

Chamoso, M., \& Rawson, W. B. (1998). ¿Hacia unas nuevas matemáticas? Salamanca, Ed. Universidad de Salamanca.

Chernyshenko, O., Stark, S., Chan, K., Drasgow, F., \& Williams, B. (2001). Fitting Item Responce Theory Models to Two Personalily Inventories: Issues and Insights. Multivariate Behavioral Research, 36(4), 523-562.

Drasgow, F., Levine, M. V., Tsien, S., Williams, B., \& Mead, A. D. (1995). Fitting polytomous item response theory models to multiple-choice tests. Applied Psychological Measurement, 19, 143-165.

Escalera-Chávez, M., García-Santillán, A., \& VenegasMartínez, F. (2014). Confirmatory factorial analysis to validity a theoretical model to measure attitude toward statistics. Mediterranean Journal of Social Science, 5(1), 569-577.

García-Santillan, A., Moreno-García, E., Castro, J., Zamudio-Abdala, J., \& Garduno-Trejo, J. (2012). Cognitive, affective and behavioral components that explain attitude toward statistics. Journal of mathematics research, 4(5), 8-16.

Gray-Little, B., Williams, V. S. L., \& Hancock, T. D.
(1997). An item response theory analysis of the Rosenberg Self-Esteem Scale. Personality and Social Psychology Bulletin, 23(5), 443-451.

González-Romá, V., \& Espejo, B. (2003). Testing the middle response categories »Not sure», «In between» and «?? in polytomous items. Psicothema, 15, 278-284.

Hernández, A., Espejo, B., \& González-Romá, V. (2006). The functioning of central categories Middle Level and Sometimes in graded response scales: Does the label matter? Psicothema, 18 (2), 300-306.

Ho, H., Senturk, D., Lam, A. G., Zimmer, J. M., Hong, S., Okamoto, Y., \& Chiu, S. (2000). The affective and cognitive dimensions of math anxiety: A crossnational study. Journal for Research in Mathematics Education, 31(3), 365-379.

Kargar, M., Tarmizi, R.A., \& Bayat, S. (2010). Relationship between mathematical thinking, mathematics anxiety and mathematics attitudes among university students. Procedia Social and Behavioral Sciences, 8(1), 537-542.

Lahuis, D., Clark, P., \& O’Brien, E. (2009). An examination of item response theory item fit índices for the graded response model. Organizational research methods, 14(1), 10-23.

Lancheros, L., Marconi, L., Manrique, M., \& Mendivelso, M. (2007). Conceptos básicos acerca de las pruebas de actitud. Avances en medición, 5,163-167.

Maravilla, J. (2006). El aprendizaje de las matemáticas en ingeniería: Una propuesta desde el paradigma constructivista psicogenético. Buenaval (invierno), 61-77.

Martinez, R. (2005). Psicometría: Teoría de los testpsicológicos y educativos. Madrid: Ed. Síntesis.

Mato-Vázquez, M. D., \& De la Torre, E. (2009). Evaluación de las actitudes hacia las matemáticas y el rendimiento académico. En M.J. González, M.T. González \& J. Murillo (Eds.), Investigación en Educación Matemática XIII (pp. 285-300). Santander: SEIEM.

Mora, J. (2010). Actitudes y Rendimiento Académico en el Área de Matemáticas de los Estudiantes que Ingresan al programa de Ingeniería en la Universidad Libre de Colombia, Sede Bogotá. 
Tesis de Maestría. Bogotá: Universidad Sergio Arboleda.

Muñiz, J. (1997). Introducción a la teoría de respuesta a los items. Madrid: Ediciones Pirámide.

National Council of Teachers of Mathematics. (2000). Principles and standards for school mathematics. Reston, VA: Author.

OECD. (2012). PISA 2009 Technical Report. Paris: OECD.

Padron, O. (2008). Actitudes hacia la matemática. Sapiens: Revista Universitaria de Investigación, 9(1), 237-256.

Pérez, L. (2008). Actitudes y rendimiento académico en matemáticas de los estudiantes que ingresan al primer semestre en la Universidad Sergio Arboleda. Tesis de Maestría. Bogotá, Universidad Sergio Arboleda.

Perlman, B., \& McCann, L. (1993). The place of mathematics and science in undergraduate psychology education. Teaching psychology, 20(4), 205-208.

Pineda, C. (2013). Validación del test de actitudes ante la estadística en una muestra de universitarios colombianos. Avances en medición, 8, 81-92.

Ponce, S., Martínez, G., \& Zuriaga, F. (2008). Creencias y Estereotipos: La dimensión afectiva y su influencia en matemática. Memorias del VI Congreso Argentino de Enseñanza de la Ingeniería (VI CAEDI). Ciudad de Salta, Argentina.

Preciado, J. (2008). Actitudes y Rendimiento Académico en Matemáticas de los Estudiantes que Ingresan por Primera vez en la Fundación Universitaria San Martin. Tesis de maestría, Departamento de ciencias. Bogotá: Universidad Sergio Arboleda.

Rhodes, E. (2014). Students and their struggles with stats. (2014). The psychologist, 27(11), 814.
Rodríguez-Jiménez, O. (2011). Análisis psicométrico de las encuestas de opinión de los estudiantes sobre la actividad docente de la Universidad Autónoma de Madrid. Trabajo de fin de máster. Madrid: Universidad Autónoma de Madrid.

Samejima, F. (1969). Estimation of latent ability using a response pattern of grades scores. Psychometrika Monograph.17, 3- 21.

Schmeiser, C. B., \& Welch, C. (2006). Test development. En: Educational Measurement, (4th Ed). Ed. R.L. Brennan. pp. 307-353. Wesport, CT: American Council on Education/Praeger.

Scientific software International, Inc., (2003). MULTILOG for Windows. [Computer program].

Schreiber, J. B., Stage, F. K., King, J., Nora, A., \& Barlow, E. A. (2006). Reporting structural equation modeling and confirmatory factor analysis results: A review. Journal of Education Research, 99, 323-337.

Stark, S. (2001). Programa MODFIT. Industrial Organizational Psychology Division. [Computer program]

Ximénez, C. (2010). Técnicas de agrupación. Documento interno. Master de Metodología en Ciencias del Comportamiento. Madrid: Universidad Autónoma de Madrid.

Yavuz Mumcu, H., \& Cansiz Aktas, M. (2015). Multiprogram high school students' attitudes and selfefficacy perceptions toward mathematics. Eurasian Journal of Educational Research, 59, 207-226.

Recibido: 04 de junio de 2015

Aceptado:14 de febrero de 2016 


\section{Anexo 1}

\section{CUESTIONARIO “ACTITUDES HACIA LAS MATEMATICAS”}

A continuación encuentra unas preguntas que tienen como fin conocer su actitud hacia las matemáticas. No tienen respuestas buenas o malas, sólo deseamos saber si Ud. está de acuerdo o en desacuerdo con cada una de las afirmaciones que se presentan, por ejemplo, ante la afirmación Me gustan las matemáticas, usted debe señalar su opinión marcando en la hoja de respuestas solo una de las siguientes alternativas:
A Si está Totalmente de Acuerdo con la afirmación.
B Si está De Acuerdo con la afirmación.
C Si No sabe o no puede responder a la afirmación.
D Si está en Desacuerdo con la afirmación.
E Si está Totalmente en Desacuerdo con la afirmación.

Asegúrese de responder a cada una de ellas con la mayor objetividad posible. Deje que su experiencia y sentimiento lo guíen para marcar la alternativa que mejor se ajuste a su valoración.

1. Las matemáticas son amenas y estimulantes para mí.

2. Disfruto con los ejercicios y problemas de matemáticas.

3. Me agrada tener que abordar muchos cursos de matemáticas.

4. Pienso que tendré que repetir algunos cursos de matemáticas.

5. Debo estudiar matemáticas si quiero ser profesional, pero las matemáticas no son mi asignatura favorita.

6. Cuando estudio matemáticas el tiempo se me hace eterno.

7. Me siento seguro al trabajar en matemáticas.

8. Estudio matemáticas porque me toca y porque me gusta.

9. La carrera que voy a estudiar tiene matemáticas, y eso me encanta.

10. Confío en poder hacer ejercicios más complicados de matemáticas.

11. Generalmente, en las clases de matemáticas, me siento física y mentalmente dispuesto y animado.

12. Prefiero estudiar cualquier otra materia en lugar de matemáticas.

13. Mi mente se pone en blanco y soy incapaz de pensar claramente cuando hago matemáticas.

14. Solo deberían enseñarse, en matemáticas, las cosas prácticas que utilizaremos como profesionales.

15. Me agradaría, si es necesario, tomar más cursos de matemáticas.

16. Estudiar matemáticas me hace perder tiempo valioso.

17. No me satisface tener que estudiar tantas matemáticas.

18. Cuando estudio matemáticas, el tiempo se me pasa muy entretenido y rápido.

19. Se me dificulta comprender y manejar el lenguaje matemático.

20. Creo poder abordar con éxito los cursos de matemáticas.

21. Las matemáticas son mi asignatura favorita. 


\section{ANEXO 2}

Función de respuesta de las categorías y función de información para los ítems
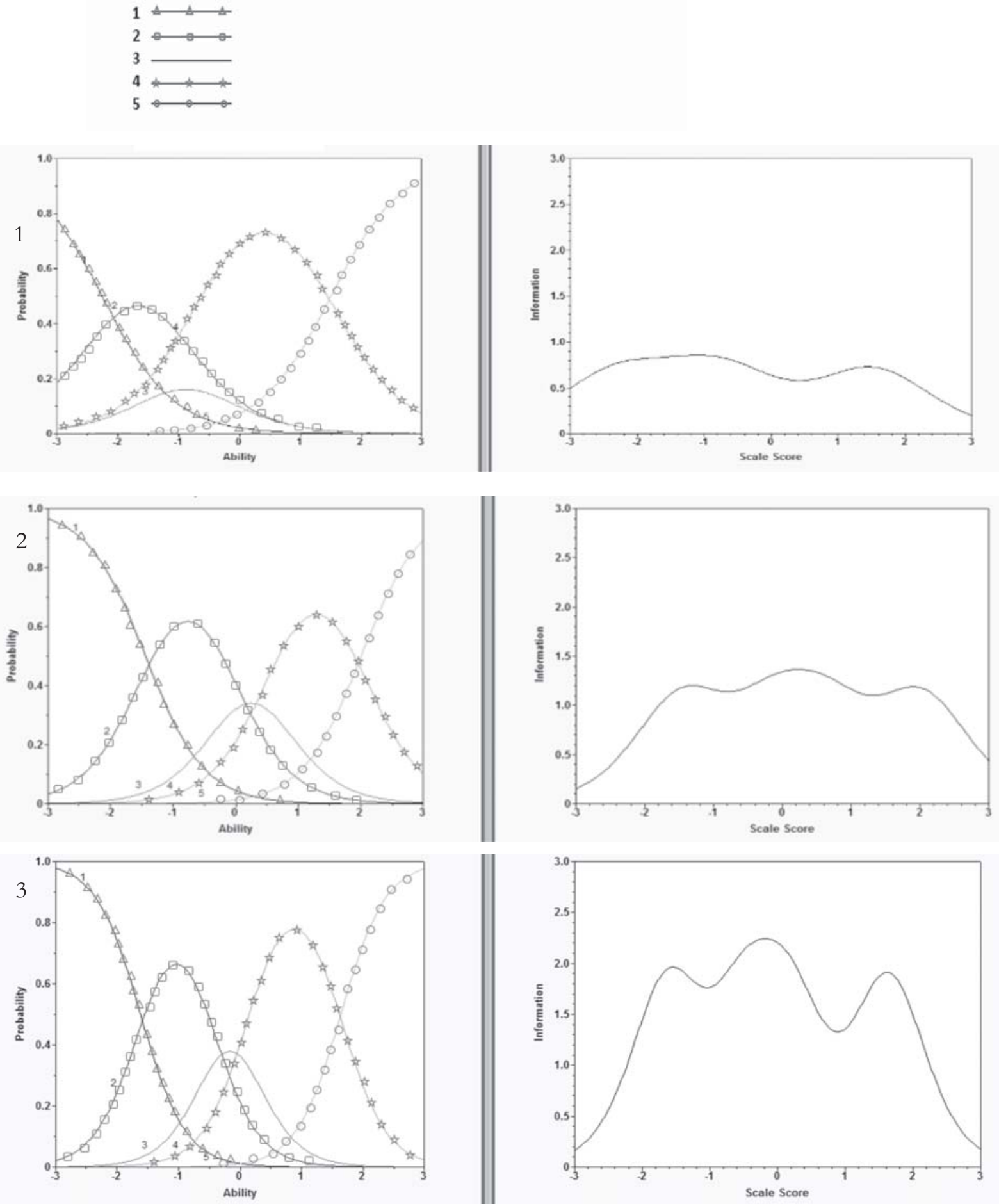

Actualidades en Psicología, 30(120), 2016, 7-30 

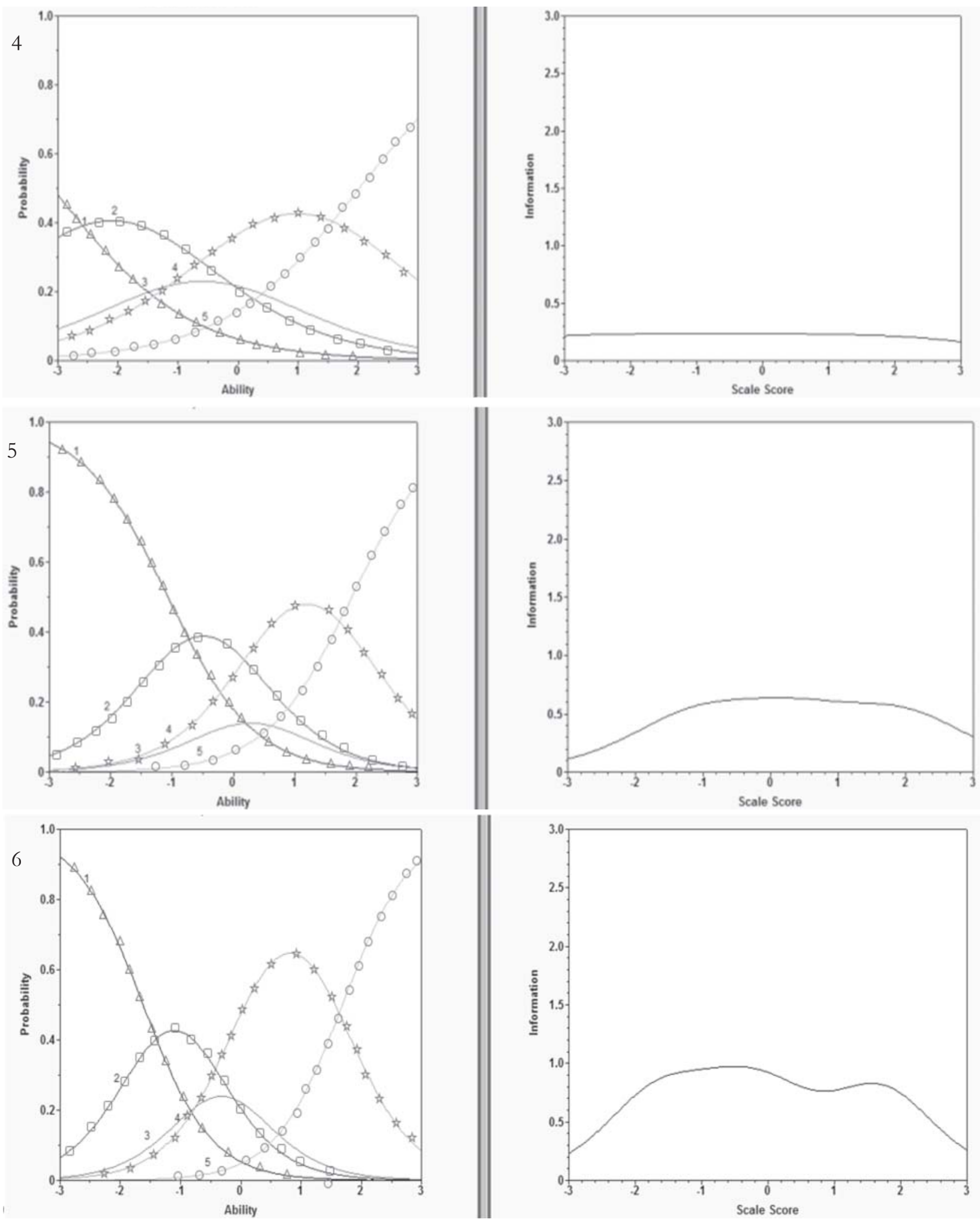

Actualidades en Psicología, 30(120), 2016, 7-30 

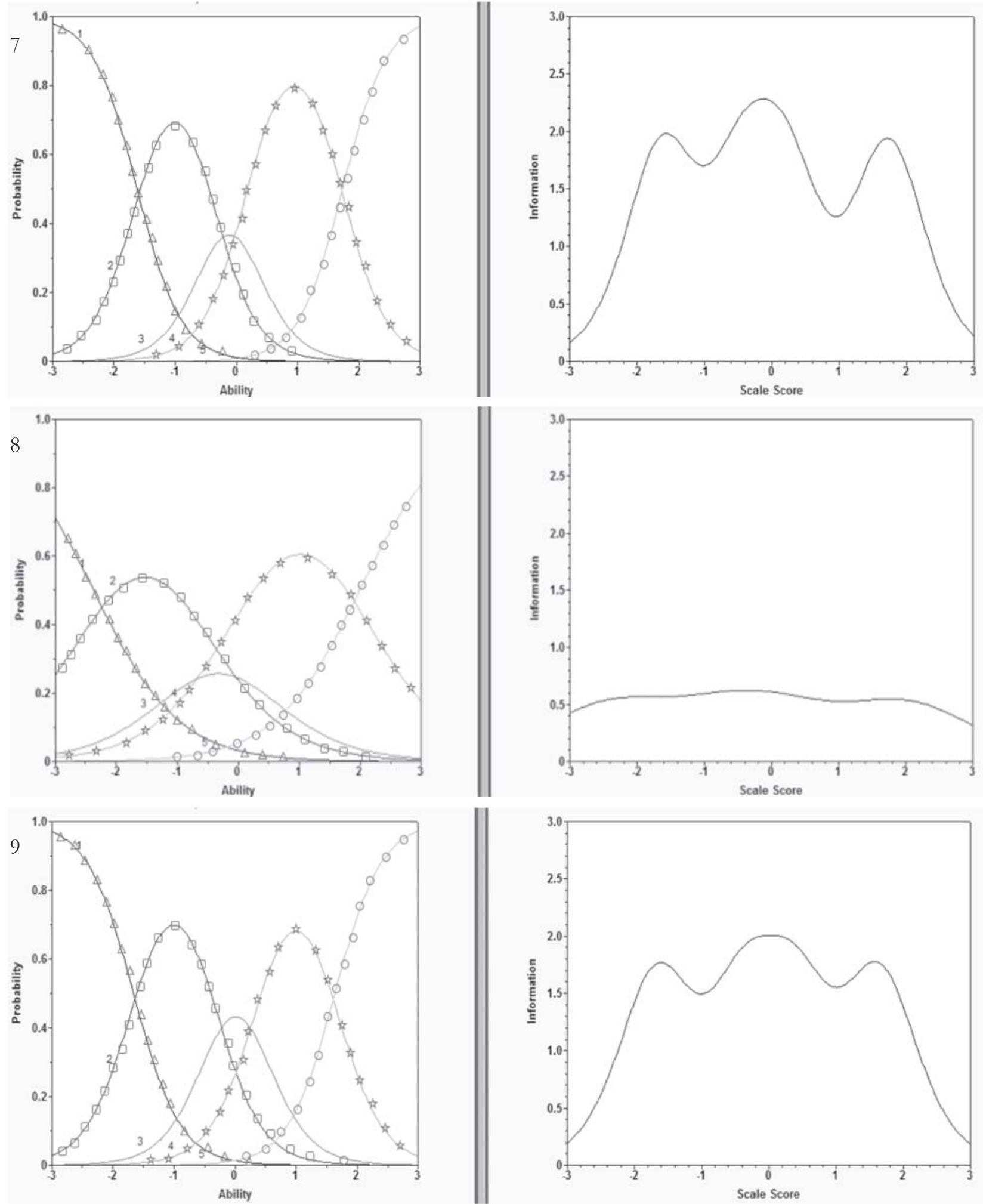

Actualidades en Psicología, 30(120), 2016, 7-30 

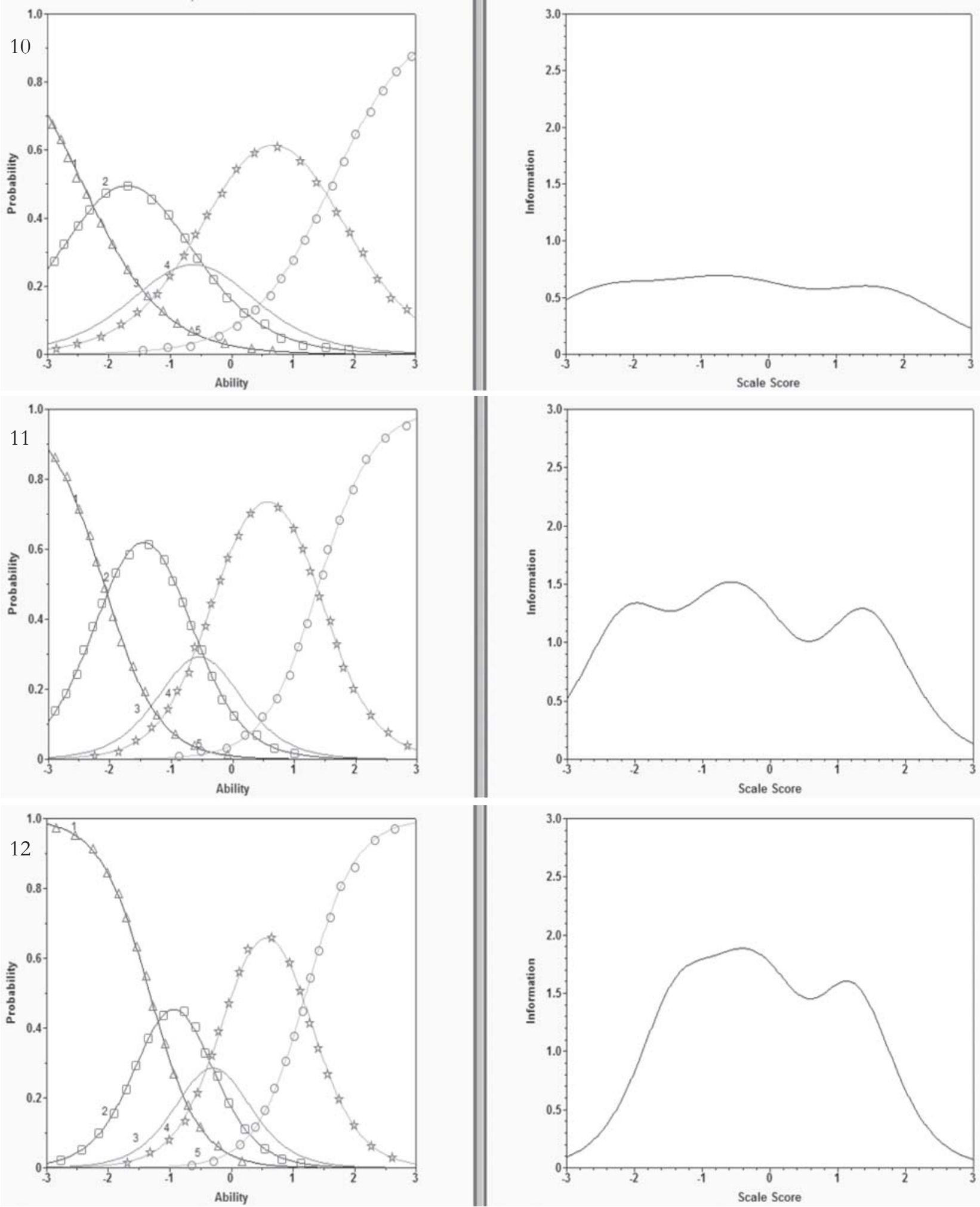

Actualidades en Psicología, 30(120), 2016, 7-30 

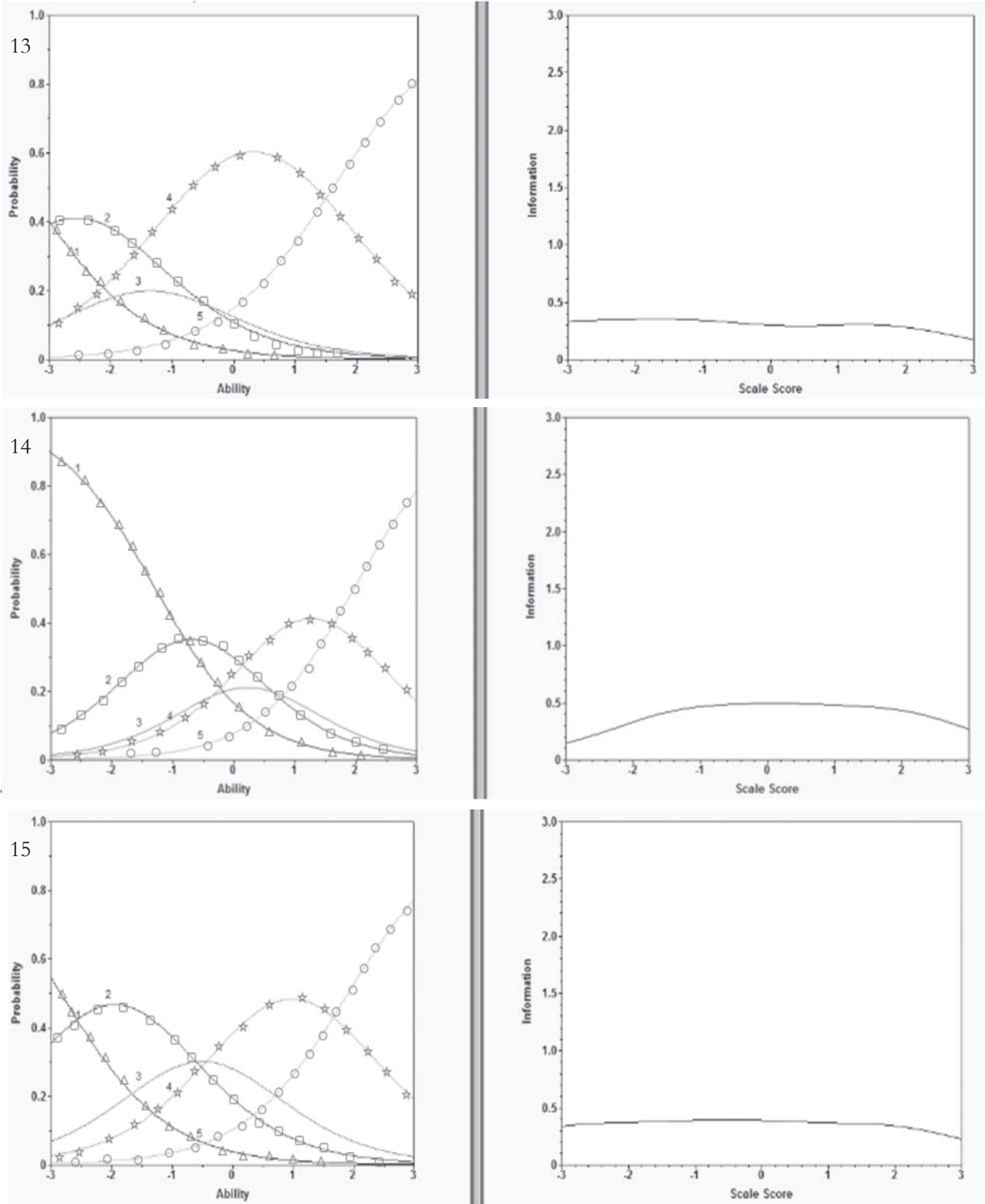

Actualidades en Psicología, 30(120), 2016, 7-30 

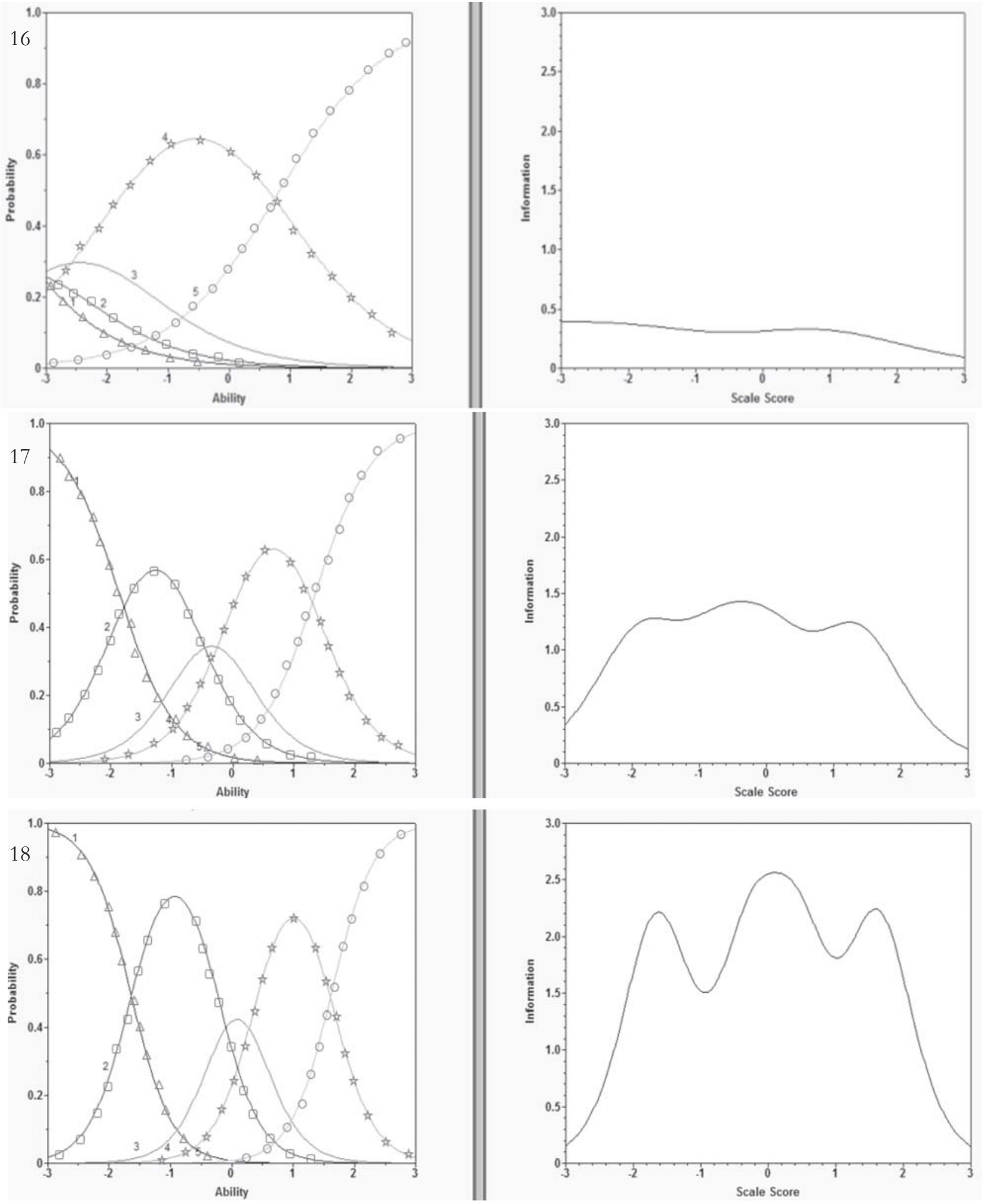

Actualidades en Psicología, 30(120), 2016, 7-30 

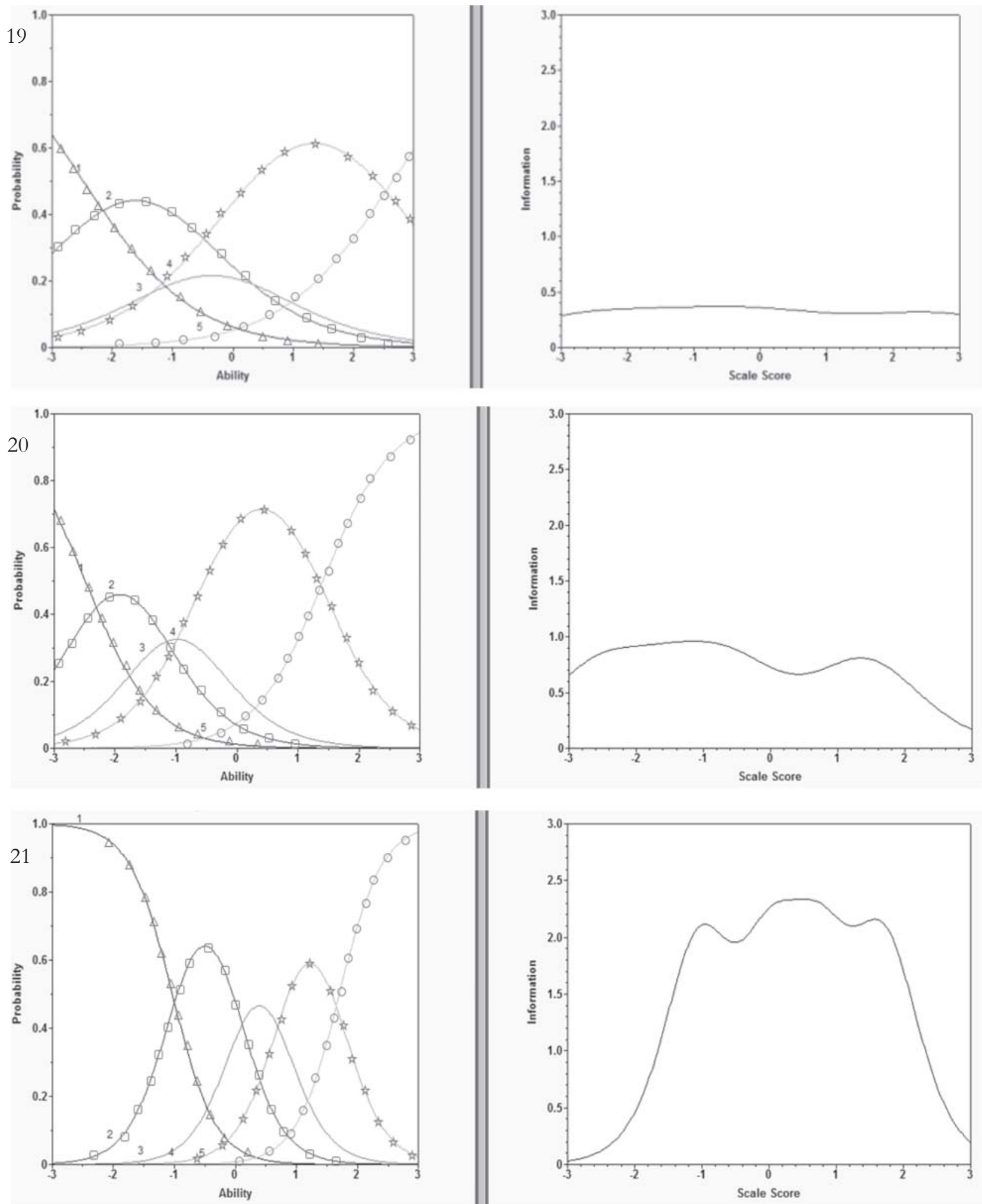

Actualidades en Psicología, 30(120), 2016, 7-30 